\title{
Magnon Modes of Microstates and Microwave-Induced Avalanche in Kagome Artificial Spin Ice with Topological Defects
}

\author{
V. S. Bhat, ${ }^{1,2, *}$ S. Watanabe $\odot,{ }^{1}$ K. Baumgaertl $\odot,{ }^{1}$ A. Kleibert, ${ }^{3}$ M. A. W. Schoen, ${ }^{3}$ C. A. F. Vaz, ${ }^{3}$ and D. Grundler $\odot^{1,4, \uparrow}$ \\ ${ }^{1}$ Institute of Materials, Laboratory of Nanoscale Magnetic Materials and Magnonics, School of Engineering, \\ École Polytechnique Fédérale de Lausanne EPFL, 1015 Lausanne, Switzerland \\ ${ }^{2}$ International Research Centre MagTop, Institute of Physics, Polish Academy of Sciences, PL-02668 Warsaw, Poland \\ ${ }^{3}$ Swiss Light Source, Paul Scherrer Institute, 5232 PSI Villigen, Switzerland \\ ${ }^{4}$ Institute of Microengineering, Laboratory of Nanoscale Magnetic Materials and Magnonics, \\ School of Engineering, École Polytechnique Fédérale de Lausanne EPFL, 1015 Lausanne, Switzerland
}

(Received 23 September 2019; revised 16 June 2020; accepted 11 August 2020; published 11 September 2020)

\begin{abstract}
We investigate spin dynamics of microstates in artificial spin ice (ASI) in $\mathrm{Ni}_{81} \mathrm{Fe}_{19}$ nanomagnets arranged in an interconnected kagome lattice using microfocus Brillouin light scattering, broadband ferromagnetic resonance, magnetic force microscopy, x-ray photoemission electron microscopy, and simulations. We experimentally reconfigure microstates in ASI using a 2D vector field protocol and apply microwave-assisted switching to intentionally trigger reversal. Our work is key for the creation of avalanches inside the kagome ASI and reprogrammable magnonics based on ASIs.
\end{abstract}

DOI: 10.1103/PhysRevLett.125.117208

Much progress has been made recently in understanding defects and disordered states in artificial spin ice [1] (ASI) - interacting ferromagnetic nanobars arranged on a two-dimensional lattice introducing geometrical frustration-via quasistatic imaging techniques [2-4]. However, their spin dynamics has been restricted to global magnetodynamics, e.g., ferromagnetic resonance (FMR), and micromagnetic simulations [5-9]. The microstates of ASIs are key also for a new type of microwave filter [6] and reprogrammable magnonic crystals [8,10-14]. Here, we report the first study using microfocus Brillouin light scattering (BLS) applied to a disordered kagome ASI. Broadband FMR and BLS spectra in major and minor loops reveal characteristic spin-wave modes which, using magnetic force microscopy (MFM) data, we directly correlate to the spin-precessional motion in individual nanomagnets within specific microstates. Further, we present a protocol to intentionally create microstates in kagome ASI, and visualize experimentally, using x-ray photoemission electron microscopy (XPEEM) [15], the onset of avalanches induced by microwave-assisted switching [16-18]. Simulations provide further microscopic insight into the magnon modes usable for reconfigurable magnonics applications.

Large (sample $A$ ) and small (samples $B$ and $C$ ) arrays of $\mathrm{Ni}_{81} \mathrm{Fe}_{19}$ nanobars were patterned on a $2 \mathrm{D}$ kagome lattice using electron beam lithography and lift-off processing. The length, width, and thickness of a given nanobar were kept at 810, 130, and $25 \mathrm{~nm}$, respectively. The $\mathrm{Ni}_{81} \mathrm{Fe}_{19}$ films of all samples were deposited simultaneously ensuring nominally identical film characteristics. The sample $A$ (for FMR), $B$ (for MFM and BLS), and
$C$ (for XPEEM) had an area of $0.7 \times 4.8 \mathrm{~mm}^{2}, 0.12 \times$ $0.12 \mathrm{~mm}^{2}$, and $0.12 \times 0.12 \mathrm{~mm}^{2}$, respectively (see Supplemental Material Fig. S1 for details [19]). The magnetization state of the kagome ASI before and after the application of microwaves to the integrated coplanar waveguide (CPW) was imaged using $\mathrm{x}$-ray magnetic circular dichroism [20]. Simulations were performed on a full bow-tie subgroup of nanobars [7,21] using the OOMMF code [22].

In Figs. 1(a) and 1(b) we show FMR spectra taken on sample $A$ with the magnetic field applied along the $x$ axis

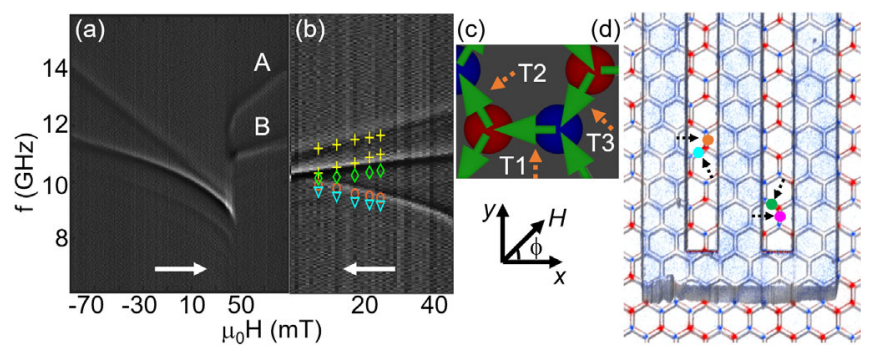

FIG. 1. Gray scale FMR spectra map of sample $A$ for a field protocol of (a) $-90 \rightarrow+90 \mathrm{mT}$ and (b) $-90 \rightarrow+45 \rightarrow 0 \mathrm{mT}$ at $\phi=0^{\circ}$. To enhance the contrast, we show the difference between neighboring spectra (derivative). White arrows represent sweep direction of $H$. (c) Definition of $T 1, T 2$, and $T 3$ nanobars with respect to the magnetic field direction. (d) Magnetic force microscopy image of sample $A$ after a field protocol of $-91.2 \rightarrow+44.1 \rightarrow 0 \mathrm{mT}$ along $\phi=0^{\circ}$ (the $x$ axis points upwards). Stray fields detected by MFM are colored in blue (red) consistent with two-in-one-out (one-in-two-out) configuration. The additional colored dots and arrows highlight positions explored by micro-BLS in Fig. 2. The integrated CPW is apparent. 
$\left(\phi=0^{\circ}\right)$ for two different field protocols. At $-90 \mathrm{mT}$, we observe two prominent branches in Fig. 1(a) corresponding to the two different groups of nanobars [Fig. 1(c)] with different resonance frequencies $f$ at $H \neq 0$ [23]; the high (low) frequency branch is attributed to $T 1$ ( $T 2$ and $T 3$ ) nanobars. With increasing $H$ the two spin-wave branches show $d f / d H<0$ (i.e., a decrease in $f$ ) up to a critical field value of $+37 \mathrm{mT}$. The negative slope indicates that the $x$ components of the magnetization vectors $\mathbf{M}$ of the nanobars do not change and are opposite to the applied magnetic field for $\mu_{0} H>0 \mathrm{mT}$ (soft mode behavior). At about $37 \mathrm{mT}$, we observe the emergence of branches $A$ and $B$ with $d f / d H>0$ indicating partial reversal of the kagome ASI. To obey $d f / d H>0$ the $x$ component of $\mathbf{M}$ of a nanobar is such that it is parallel to $\mathbf{H}$ [23]. At $48 \mathrm{mT}$, the reversal is complete, and the branches $A$ and $B$ are both prominent for further increasing $H$. We attribute the range between 37 and $48 \mathrm{mT}$ to the transition region of the kagome ASI in which switching (reversal) of nanobars occurs and disordered magnetic states are present. To study the dynamics with FMR inside the transition regime, we applied a specific magnetic field history (minor loop). We ramped the field from $-90 \mathrm{mT}$ to $\mu_{0} H_{\mathrm{pr}}=+45 \mathrm{mT}$ and then recorded spectra for the decreasing field as shown in Fig. 1(b). Here, three prominent branches are present: $A$ and $B$ with $d f / d H>0$ as well as $C$ with $d f / d H<0$. In the minor loop, we do not see abrupt changes in the branches, suggesting that the disordered state of the kagome ASI remains magnetically stable. Branch $C$ is attributed to nanobars that have not yet changed their original magnetization orientation and therefore display a soft mode behavior with increasing opposing $H>0$. The characteristics will be studied in detail in sample $B$ by local microscopy below.

The stray-field distribution (measured by MFM) of sample $B$ in the fully magnetized state exhibits two-inone-out vertices (blue) surrounded by two-out-one-in (red) vertices (not shown). Subsequently, we applied the magnetic field history of $\mu_{0} H_{\mathrm{pr}}=+44.1 \mathrm{mT} \rightarrow 0 \mathrm{mT}$ and found an irregular distribution of blue and red vertices [Fig. 1(d)]. After monitoring the remnant magnetic configuration of sample $B$ shown in Fig. 1(d) we performed microfocus BLS in applied fields $H$ which allowed us to identify the orientation of $\mathbf{M}$ of individual nanobars. Spectra taken as a function of $H$ for $H<H_{\mathrm{pr}}$ in the center of four different nanobars are displayed in Figs. 2(a)-2(d). The applied fields were below the starting field of the transition region. The cartoon image in Fig. 2(e) specifies the investigated nanobars and how they were embedded in microstates. Each spectrum in Figs. 2(a)-2(d) shows several resonances of different signal strength. With increasing $H$ the different resonance peaks shift consistently to either smaller [Figs. 2(a) and 2(c)] or larger [Figs. 2(b) and 2(d)] frequency values. The resonance frequencies and slopes $d f / d H$ extracted from BLS are

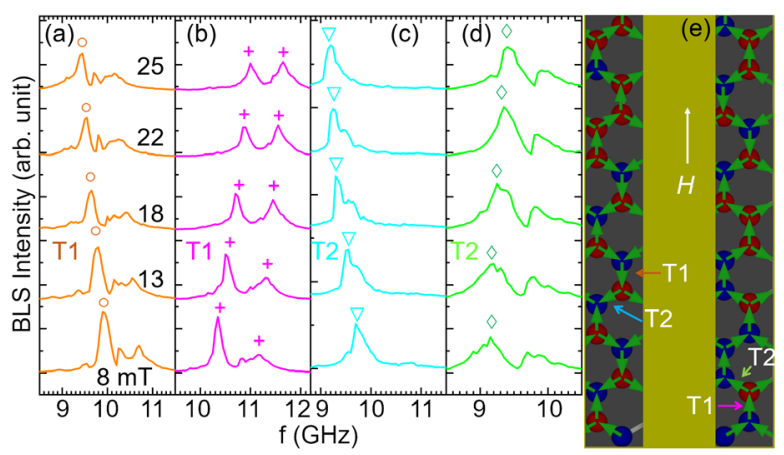

FIG. 2. BLS intensities measured for different external field values when the laser spot was placed on the centers of nanobars indicated by (a) orange, (b) magenta, (c) cyan, and (d) green filled circles in Fig. 1(d). We observe resonance peaks obeying $d f / d H<0$ in (a) and (b), and $d f / d H>0$ for (c) and (d). The resonance frequencies highlighted by symbols in (a) to (d) are overlaid on FMR data in Fig. 1(b). (e) Representative sketch illustrating charges on vertices (colored filled circles) and magnetization directions (bold arrows) of the kagome ASI extracted near the CPW (light green) from combined analysis of MFM and BLS data, respectively (see Supplemental Material Fig. S2 for the complete map [19]). The colors of arrows highlighting studied nanobars are consistent with spectra shown in (a) to (d).

consistent with the spectra obtained on sample $A$ [symbols in Fig. 1(b)]. The BLS data now allow us to understand microscopically the origin of the branches detected via broadband FMR. From MFM data and $d f / d H$ measured by BLS on the individual nanobars of the kagome ASI we extract the orientation of the $x$ component of their magnetization $\mathbf{M}$ and display the corresponding vectors $\mathbf{M}$ (bold green arrows) in Fig. 2(e). Here, we incorporate also the magnetic pole distribution of Fig. 1(d) found by MFM. This combined map allows us to identify magnetization orientation of nanobars and their different magnetic surrounding [4] (see Fig. S2 in the Supplemental Material for the complete MFM and BLS magnetization map [19]).

Different approaches have been utilized to understand the magnetization reversal in the kagome ASI [4,24]. Based on Fig. 2(e) we classify five types of nanobar configurations $(C)$ which we investigate further in Fig. 3: $C 1$ corresponds to the reference configuration [Fig. 3(a)], where vectors $\mathbf{M}$ of all the $T 1$ and $T 2$ nanobars have a $-x$ component. The configurations $C 2, C 3, C 4$, show three, four, and four out of five nanobars switched, respectively; configuration $C 5$ represents completely reversed nanobars. Configurations $C 2$ and $C 5$ both contain switched $T 1$ nanobars, but have different immediate surrounding or topological defect configuration.

In Fig. 3(b) BLS spectra taken on $C 1$ to $C 5$ at $25 \mathrm{mT}$ are shown. For these spectra the laser was focused on the center of each central $T 1$ nanobar. All spectra contain at least one prominent mode. The spectrum of $C 1$ is consistent with the spectrum at $25 \mathrm{mT}$ in Fig. 3(a) taken on a different 


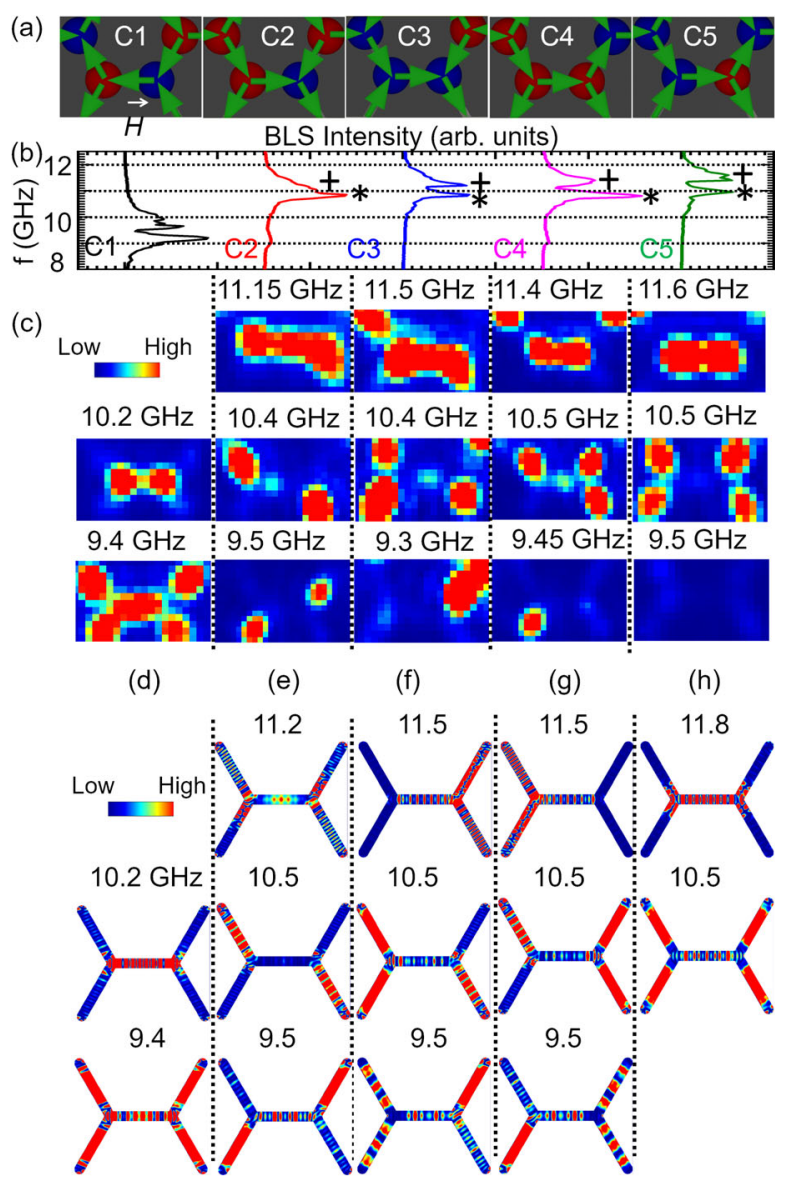

FIG. 3. (a) Sketches of charge configurations (blue and red spheres) and magnetization vectors $\mathbf{M}$ (arrows) for configurations $C 1$ to $C 5$. (b) BLS intensities measured at the central position on $T 1$ nanobars belonging to the configurations displayed above the spectra at $\mu_{0} H=25 \mathrm{mT}$. (c) Spatially resolved BLS intensity maps at $25 \mathrm{mT}$ for fixed frequencies in configurations $C 1$ to $C 5$ displayed above. Here, red (blue) color corresponds to maximum (minimum) BLS intensity. (d)-(h) Simulated spatial distribution of power absorption in $T 1$ and $T 2$ nanobars for configurations $C 1$ to $\mathrm{C} 5$ at $\mu_{0} H=25 \mathrm{mT}$. Red (blue) color corresponds to $0.2 \mathrm{k}(\mathrm{A} / \mathrm{m})^{2}\left[0 \mathrm{k}(\mathrm{A} / \mathrm{m})^{2}\right]$ reflecting spin-precessional amplitude $M_{z}^{2}$.

$T 1$ nanobar experiencing a similar $C 1$ configuration. This substantiates the reproducibility of observed resonances. Configurations $C 3$ to $C 5$ give rise to a clearly developed double peak resonance. Comparing $C 1$ [left in Fig. 3(b)] and $C 5$ (right) their resonance peaks display the largest overall discrepancy in frequency. This is expected from the different magnetic orientations of the central $T 1$ nanobars [23]. The resonance peaks of spectra of $C 2$ to $C 4$ reside at slightly smaller frequencies compared to $C 5$ though their central $T 1$ nanobars exhibit the same orientation of $\mathbf{M}$ : the most prominent resonance of $C 2, C 3$, and $C 4$ (marked by asterisks) is found at about $10.8 \mathrm{GHz}$ instead of $11.0 \mathrm{GHz}$ observed for $C 5$. The resonance features of $C 2$ to $C 5$ at frequencies $f>11.0 \mathrm{GHz}$ (marked by crosses) are found to vary from configuration to configuration.
By means of scanning BLS [Fig. 3(c)] we find that also the magnon mode patterns in the complete bow-tie subgroup of the ASI vary significantly depending on the topological defect configuration; for $f>11 \mathrm{GHz}$ (top row), i.e., excitation at resonance frequencies marked by crosses in Fig. 3(b), the central $T 1$ nanobar is prominently excited for $C 2$ to $C 5$. The same mode pattern is found also for excitation at the resonances marked by asterisks in Fig. 3(b) (not shown). Between 10.4 and $10.5 \mathrm{GHz}$ (shown in the central row) the central $T 1$ nanobars of $C 2$ to $C 5$ are much less excited. Instead, specific $T 2$ nanobars show resonant excitation depending on the configuration of the topological defects. Near $9.5 \mathrm{GHz}$ [bottom row in (c)] configuration $C 1$ exhibits pronounced spin precession in the complete bow-tie subgroup (left). This behavior is contrary to $C 2$ to $C 5$ for which the central $T 1$ nanobars are not excited and $T 2$ nanobars show spatial excitation patterns which are strikingly complementary to the patterns found between 10.4 and $10.5 \mathrm{GHz}$ (shown in the central row). These observations evidence that, consistent with predictions in Ref. [7], topological defects modify eigenfrequencies of nanobars in kagome ASIs in a specific manner (see Supplemental Material Figs. S3-S7 for more spatially resolved maps at various frequencies in configurations $C 1-C 5$ [19]).

The micromagnetic simulations are shown in Figs. 3(d)-3(h). Overall we find a good agreement between simulations and experimentally observed characteristics. Some simulated power maps suggest spatially varying intensities (fine structures) of spin-precessional motion inside nanobars. We are not able to resolve them with BLS due to its limited spatial resolution of about $300 \mathrm{~nm}$. The complementary mode patterns detected at frequencies near 9.5 and $10.5 \mathrm{GHz}$ in $T 2$ nanobars of configurations $C 2, C 3$, and $C 4$ are well reproduced by the simulations. The latter indicate that we detect the uniform excitation of corresponding nanobars. The agreement is also found for the mode patterns of $C 1$ suggesting that simulations performed on the bow-tie subgroup allow us to explore the effect of topological defect configurations.

$T 2$ nanobars show peculiar characteristics: (i) in case of a negative $x$ component of $\mathbf{M}$ the bars show almost uniform excitation at a low frequency of $9.5 \mathrm{GHz}$; (ii) when all three nanobars connected to a single vertex have undergone switching to the applied field direction, the switched $T 2$ nanobars exhibit a nonuniform spin-wave mode at 9.5 GHz. It reflects a standing backward volume magnetostatic spin wave [bottom row of Figs. 3(f) and 3(g)]. The same $T 2$ nanobars are uniformly excited at $10.5 \mathrm{GHz}$ [central row in Figs. 3(f) and 3(g)]. The topological defect configuration clearly modifies the spin excitations in $T 2$ nanobars and converts them from a uniform mode at 9.5 GHz [compare bottom panel in Fig. 3(d)] to a standing spin wave; consistently FMR spectra obtained on the fully saturated kagome ASI contain such standing spin-wave 
modes [faint branch below mode $B$ in Fig. 1(a)]; (iii) if a switched $T 2$ nanobar is connected to a vertex where only one other nanobar has been reversed, the switched $T 2$ nanobar does not support pronounced excitation at $9.5 \mathrm{GHz}$ [lower row in Figs. 3(e)-3(g)]. Discrepancies remain for predicted mode frequencies in configuration $\mathrm{C}_{5}$ and for excitations beyond about $11.5 \mathrm{GHz}$. We speculate that a homogeneously magnetized surrounding ASI provides an additional biasing magnetic field that is not contained in the simulations and modifies eigenfrequencies.

The realization of tailored microstates is crucial for both reconfigurable magnonic crystals $[12,14,25,26]$ as well as microwave-induced avalanches (Dirac strings) triggered inside the kagome ASI [7]. We employed a twodimensional magnetic field protocol on sample $A$ aiming at an intentionally reconfigured kagome ASI. For a positive magnetic field applied at an angle of $\phi=20^{\circ}$, we divide the kagome ASI into three types of nanobars concerning their misalignment angles with respect to the applied field $H$ (angles given in brackets): $T 1\left(20^{\circ}\right), T 2\left(40^{\circ}\right)$, and $T 3\left(80^{\circ}\right)$ [labels are defined in Fig. 1(c)]. As we sweep the field from +90 to $0 \mathrm{mT}$ along $\phi=20^{\circ}$, we see the branches $A, B 1$, and $B 2$ (corresponding to the nanobars $T 1, T 2$, and $T 3$, respectively) with different $d f / d H$ (T3 is magnetized in the hard-axis direction). At $-37 \mathrm{mT}$ two branches appear at 11.3 and $12 \mathrm{GHz}$ attributed to switched $T 1$ (red) and $T 2$ (blue) nanobars, respectively. These two branches $A$ and $B 1$, respectively, change their slopes at $-77 \mathrm{mT}$ for further decreasing $\mu_{0} H$. At the same field, we also see the reappearance of branch $B 2$ belonging to $T 3$ (green) nanobars which is mirrored with respect to the large positive $H$. This observation indicates that $\mathbf{M}$ of $T 3$ nanobars is opposed to $\mu_{0} \mathbf{H}$ between -37 and $-77 \mathrm{mT}$ whereas $\mathbf{M}$ vectors of $T 1$ and $T 2$ nanobars are aligned with $\mathbf{H}$ [black arrow at the top of Fig. 4(a)]. We label the corresponding bow-tie microstate in this intermediate field regime by configuration $C 2^{\prime}$ in Fig. 4(a). It resides between the configurations $C 1$ and $C 5$.

We now demonstrate how to reconfigure the kagome ASI from configuration $C 2^{\prime}$ to $C 2$ [sketched on the top of Fig. 4(b)]. We first sweep the field from $\mu_{0} H=90 \mathrm{mT}$ at $\phi=20^{\circ}$ to $-76 \mathrm{mT}$ and then to $0 \mathrm{mT}$ at the same angle $\phi$. Up to $0 \mathrm{mT}$ the branches are monotonic indicating that $C 2^{\prime}$ is stable as the remnant configuration. At $0 \mathrm{mT}$ we change the angle setting to $\phi=-20^{\circ}$. Subsequently, we increase the field from 0 to $90 \mathrm{mT}$ along $\phi=-20^{\circ}$. At $\phi=-20^{\circ}$, the $\mathbf{M}$ vectors of $T 1$ (red) and $T 2$ (blue) nanobars are opposed to $\mathbf{H}$ whereas $T 3$ (green) nanobars of configuration $C 2^{\prime}$ are aligned. Therefore, only these latter nanobars lead to a branch with $d f / d H>0$ while the other two types of nanobars induce branches with $d f / d H<0$. At about $35 \mathrm{mT}$ in Fig. 4(b) we see that a high-frequency branch $A$ corresponding to switched $T 1$ (red) nanobars shows up. Because of the smaller misalignment angle with $\mathbf{H}$ their resonance is at a higher frequency than the branch of the $T 3$

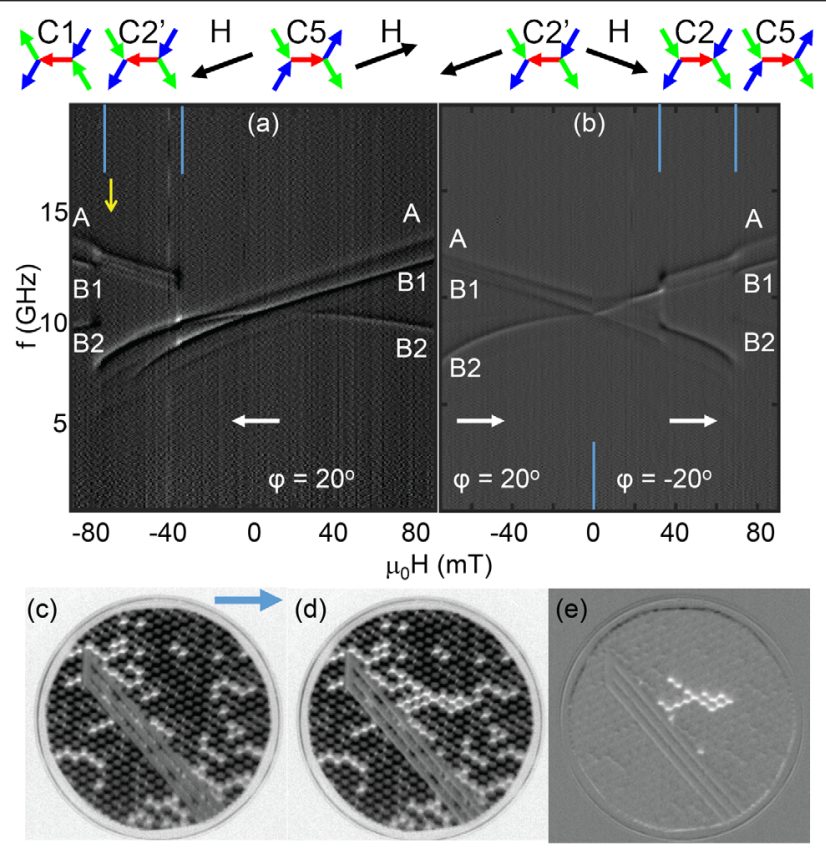

FIG. 4. Gray scale FMR spectra of sample $A$ for field protocols of (a) a major loop with $90 \rightarrow-90 \mathrm{mT}$ at $\phi=20^{\circ}$ and (b) after first applying $90 \rightarrow-76 \mathrm{mT}$ [yellow arrow in (a)], a minor loop with $-76 \rightarrow 0 \mathrm{mT}$ at $\phi=20^{\circ}$ and then $0 \rightarrow+90 \mathrm{mT}$ at $\phi=-20^{\circ}$. The solid white arrows indicate field sweep directions. We show the difference between neighboring spectra (derivative). The dashed blue colored lines highlight regimes attributed to particular microstate configurations shown on the top. The black arrows indicate field directions with respect to the bow-tie subgroup. XPEEM images taken at remanence (c) before and (d) after applying a microwave signal at $9.3 \mathrm{GHz}$. The magnetization of white (black) colored nanobars points along (opposite to) the magnetic field which was applied under microwave irradiation. The CPW appears in light-gray color. (e) Difference between XPEEM images shown in (c) and (d). Nanobars switched by microwaves are shown in white color. The blue solid arrow indicates the x-ray and magnetic field direction.

nanobars (labeled by $B 1$ ). These latter nanobars do not change their magnetization direction for increasing field. We call the new configuration in the bow-tie subgroup $C 2$. At $70 \mathrm{mT}$, switching of $T 2$ nanobars occurs and configuration $C 5$ is reached. Note that $C 5$ is the mirrored configuration of $C 1$ such that the observed resonance frequencies (branches) are identical to the ones at $\mu_{0} H<$ $-80 \mathrm{mT}$ for $\phi=20^{\circ}$ shown in (a). In the intermediate field regime between 35 and $70 \mathrm{mT}$ (configuration $C 2$ ) only the central $T 1$ nanobar-out of five nanobars of the bow-tie subgroup - is oriented differently compared to $C 2^{\prime}$. Thus, the presented two-dimensional field protocol allows one to reconfigure nanobars systematically and utilize their distinctly different spin-wave spectra for reconfigurable magnonic crystal applications.

To create nanobar reversal within kagome ASI, we utilized the microwave-assisted switching scheme proposed in Ref. [7] and performed it inside an ultra-high-vacuum 
XPEEM chamber for magnetic imaging (see Supplemental Material Fig. S8 [19]). We first prepared the magnetic configuration displayed in Fig. 4(c) by applying a small positive magnetic field after saturation at large negative $H$. Then we applied a microwave signal with the resonance frequency of configuration $C 1$ in the same opposing field and took an XPEEM image at remanence [Fig. 4(d)]. The white-colored nanobars showing up in the difference signal [Fig. 4(e)] are the ones switched due to microwave-assisted switching. They form a complex string which is about $9 \mu \mathrm{m}$ long and indicates the intended avalanchelike reversal. Our observation highlights that two-dimensional ASIs allow for microwave-assisted gating of spin-wave propagation over macroscopic distances in contrast to the local processes reported in Refs. [16-18,27].

In summary, we performed global FMR and local BLS spectroscopy on kagome ASI. Combined with MFM data, the BLS technique showed that topological defects modified microscopically the magnon mode patterns in the bow-tie subgroup, particularly for low frequency excitations (here for $f \leq 10.5 \mathrm{GHz}$ ). We demonstrated experimentally both the intentional generation of microstates with different $\mathrm{GHz}$ spin dynamics and the microwave-assisted switching of strings inside an ASI. Our work opens up routes for reconfigurable magnonics based on tailored microstates in kagome ASI.

Data can be downloaded from zenodo via [28].

The research was supported by the Swiss National Science Foundation via Grant No. 163016. V. S. B. acknowledges support from the foundation for Polish Science through the IRA Programme financed by EU within SG OP Programme. Part of this work was performed at the Surface/Interface Microscopy (SIM) beam line of the Swiss Light Source (SLS), Paul Scherrer Institut (PSI), Villigen Switzerland.

*vbhat@magtop.ifpan.edu.pl

†dirk.grundler@epfl.ch

[1] R. F. Wang, C. Nisoli, R. S. Freitas, J. Li, W. McConville, B. J Cooley, M. Lund, N. Samarth, C. Leighton, V. H. Crespi et al., Nature (London) 439, 303 (2006).

[2] Y. Qi, T. Brintlinger, and J. Cumings, Phys. Rev. B 77, 094418 (2008).

[3] S. Ladak, D. E. Read, G. K. Perkins, L. F. Cohen, and W. R. Branford, Nat. Phys. 6, 359 (2010).

[4] E. Mengotti, L. J. Heyderman, A. F. Rodríguez, F. Nolting, R. V. Hügli, and H.-B. Braun, Nat. Phys. 7, 68 (2011).

[5] S. Gliga, A. Kákay, R. Hertel, and O. G. Heinonen, Phys. Rev. Lett. 110, 117205 (2013).

[6] X. Zhou, G.-L. Chua, N. Singh, and A. O. Adeyeye, Adv. Funct. Mater. 26, 1437 (2016).
[7] V. S. Bhat, F. Heimbach, I. Stasinopoulos, and D. Grundler, Phys. Rev. B 93, 140401(R) (2016).

[8] M. B. Jungfleisch, W. Zhang, E. Iacocca, J. Sklenar, J. Ding, W. Jiang, S. Zhang, J. E. Pearson, V. Novosad, J. B. Ketterson et al., Phys. Rev. B 93, 100401(R) (2016).

[9] V.S. Bhat, F. Heimbach, I. Stasinopoulos, and D. Grundler, Phys. Rev. B 96, 014426 (2017).

[10] L. J. Heyderman and R. L. Stamps, J. Phys. Condens. Matter 25, 363201 (2013).

[11] M. Krawczyk and D. Grundler, J. Phys. Condens. Matter 26, 123202 (2014).

[12] D. M. Arroo, J. C. Gartside, and W. R. Branford, Phys. Rev. B 100, 214425 (2019).

[13] S. H. Skjærvø, C. H. Marrows, R. L. Stamps, and L. J. Heyderman, Nat. Rev. Phys. 2, 13 (2019).

[14] S. Gliga, E. Iacocca, and O. G. Heinonen, APL Mater. 8, 040911 (2020).

[15] C. A. F. Vaz, A. Kleibert, and M. E. Kazzi, in 21st Century Nanoscience-A Handbook: Advanced Analytic Methods and Instrumentation, edited by K. D. Sattler (CRC Press, Boca Raton, 2020), Vol. 3.

[16] C. Thirion, W. Wernsdorfer, and D. Mailly, Nat. Mater. 2, 524 (2003).

[17] J. Podbielski, D. Heitmann, and D. Grundler, Phys. Rev. Lett. 99, 207202 (2007).

[18] H. T. Nembach, P. Martín Pimentel, S. J. Hermsdoerfer, B. Leven, B. Hillebrands, and S. O. Demokritov, Appl. Phys. Lett. 90, 062503 (2007).

[19] See Supplemental Material at http://link.aps.org/ supplemental/10.1103/PhysRevLett.125.117208 further SEM and MFM images along with the obtained magnetization map determined using BLS and MFM data, spatially resolved BLS intensity maps at various frequencies, an image of the printed circuit board with a voltage controlled oscillator and further XPEEM images showcasing microwave-assisted switching.

[20] L. Le Guyader, A. Kleibert, A. F. Rodríguez, S. El Moussaoui, A. Balan, M. Buzzi, J. Raabe, and F. Nolting, J. Electron Spectrosc. Relat. Phenom. 185, 371 (2012).

[21] P. Mellado, O. Petrova, Y. Shen, and O. Tchernyshyov, Phys. Rev. Lett. 105, 187206 (2010).

[22] M. Donahue and D. Porter, Interagency Report No. NISTIR 6376 (1999).

[23] A. G. Gurevich and G. A. Melkov, Magnetization Oscillations and Waves (CRC Press, Boca Raton, 1996).

[24] P. Mellado, Ph.D. Thesis, The Johns Hopkins University, 2010.

[25] S. Lendinez and M. B. Jungfleisch, J. Phys. Condens. Matter 32, 013001 (2019).

[26] A. Talapatra, N. Singh, and A. O. Adeyeye, Phys. Rev. Applied 13, 014034 (2020).

[27] A. Haldar and A. O. Adeyeye, Appl. Phys. Lett. 116, 162403 (2020).

[28] V. S. Bhat, S. Watanabe, K. Baumgaertl, A. Kleibert, M. A. W. Schoen, C. A. F. Vaz, and D. Grundler, https:// doi.org/10.5281/zenodo.3997242. 


\title{
Supplemental Material for Magnon Modes of Microstates and Microwave-Induced Avalanche in Kagome Artificial Spin Ice with Topological Defects
}

\author{
V.S. Bhat ${ }^{1,2}$, S. Watanabe ${ }^{1}$, K. Baumgaertl ${ }^{1}$, A. Kleibert ${ }^{3}$, M. A. W. Schoen ${ }^{3}$, C.A.F. Vaz ${ }^{3}$, and D. Grundler ${ }^{1,4 *}$ \\ ${ }^{1}$ Institute of Materials, Laboratory of Nanoscale Magnetic Materials and Magnonics, \\ School of Engineering, École Polytechnique Fédérale de Lausanne, EPFL, 1015 Lausanne, Switzerland; \\ ${ }^{2}$ International Research Centre MagTop, Institute of Physics, \\ Polish Academy of Sciences, PL-02668 Warsaw, Poland, \\ 3 Swiss Light Source, Paul Scherrer Institute, 5232 PSI Villigen Switzerland, \\ ${ }^{4}$ Institute of Microengineering, Laboratory of Nanoscale Magnetic Materials and Magnonics, \\ School of Engineering, École Polytechnique Fédérale de Lausanne, EPFL, 1015 Lausanne, Switzerland
}

(Dated: August 24, 2020)

The document contains further SEM and MFM images along with obtained magnetization map determined using BLS and MFM data. We show spatially resolved BLS intensity maps at various frequencies. We further provide the image of a printed circuit board with a voltage controlled oscillator used in the ultra-high vacuum chamber and XPEEM images showcasing microwave-assisted switching.

https://journals.aps.org/prl/abstract/10.1103/PhysRevLett.125.117208

Copyright by American Physical Society 2020 


\section{Sample Details}

Large (Sample-A) and small (Sample-B and C) arrays of $\mathrm{Ni}_{81} \mathrm{Fe}_{19}$ nanobars were patterned on a $2 \mathrm{D}$ kagome lattice using electron beam lithography and lift-off processing. The length, width, and thickness of a given nanobar were kept at 810, 130, and $25 \mathrm{~nm}$, respectively. The $\mathrm{Ni}_{81} \mathrm{Fe}_{19}$ films of all samples were deposited simultaneously ensuring nominally identical film characteristics. The FMR was performed on Sample-A [Fig.S1(a) and (b)] (with an area of $\left.0.7 \times 4.8 \mathrm{~mm}^{2}\right)$ using a coplanar waveguide $(\mathrm{CPW})$ with a $20 \mu \mathrm{m}$ wide signal line. The MFM and BLS measurements were performed on the nanobars of Sample-B (covering an overall area of $0.12 \times 0.12 \mathrm{~mm}^{2}$ ) using an integrated CPW with a $2 \mu \mathrm{m}$ wide signal line (Fig. S1(c)). Applying a micro-focus configuration with a laser of a wavelength of 473 $\mathrm{nm}$, BLS provided a spatial resolution of about $300 \mathrm{~nm}$. For Sample-C (with an area of $0.7 \times 4.8 \mathrm{~mm}^{2}$ ) the CPW signal-line width was reduced to $800 \mathrm{~nm}$ to maximize the radio-frequency magnetic field and oriented at $\phi=45^{\circ}$ to maximize the number of nanobars under the signal line.

\section{Experimental Protocol for Studying the Magnetic Microstates}

Our experimental protocol towards studying spin dynamics in the disordered regime was as follows: (I) Broadband FMR on Sample-A in different magnetic states (major and minor loops) addressing the overall dynamic response in saturated and disordered states. (II) Application of a magnetic field $H$ of $-91.2 \mathrm{mT}$ to saturate Sample-B along the $-x$-direction. (III) MFM on the remnant state of Sample-B after saturation. (IV) Application of $\mu_{0} H_{\mathrm{pr}}=+44.1 \mathrm{mT}$ inducing a partial reversal (pr) and magnetically disordered state of Sample-B. (V) MFM of this state at $H=0[$ Fig. $1(\mathrm{~d})$ in the main text]. (f) Micro-focus BLS on the disordered state of Sample-B at various fields $H<H_{\mathrm{pr}}($ Fig. 3 in the main text).

\section{Microwave-Assisted Switching Experimental Protocol}

To perform the microwave-assisted switching we needed to find a way to introduce a local microwave signal compatible with the XPEEM measurement conditions, including ultra-high-vacuum. This was achieved with the help of a voltage -controlled-oscillator (VCO) [Fig. S8(a)]. The choice of a VCO depended on the RF output power and the microwave frequency under consideration. From the FMR and BLS data shown in Fig. 1 and 3 of the main text, the frequency of nanobars before switching is in the vicinity of $9.3 \mathrm{GHz}$. Before, we preformed the microwave-assisted switching, we carried out the DC magnetization reversal studies on our samples using XPEEM. This allowed us to find out input current value, $I$ (that generated the DC magnetic field) needed to bring kagome ASI into disordered regime. Our experimental protocol for the microwave assisted switching in KASI was as follows: (1) Apply negative maximum field (corresponding to the input current $I=-3.0 \mathrm{~A}$ ) and image the sample at $I_{o}=0$ A. (2) Change the polarity of magnetic field at $I_{o}=0 \mathrm{~A}$, and move towards positive field direction. (3) Reach a given value of the current $I_{1}$ and image the sample at $I_{o}$ [Fig. S8(b)]. (3) Reach DC magnetic field value by ramping up to the $I_{1}$ $20 \mathrm{~mA}$. (4) Apply a particular control voltage of the VCO $(+9 \mathrm{~V}$ and $+10 \mathrm{~V}$ corresponding to frequency between $9.2 \mathrm{GHz}$ and $9.3 \mathrm{GHz}$ ). (5) Turn off the VCO and ramp down the current to $I_{o}=0$ A. (6) Image the sample at the remnant state $I_{o}=0 \mathrm{~A}[$ Fig. $\mathrm{S} 8(\mathrm{c})]$.

\section{OOMMF Simulation Details}

The $\mathrm{Ni}_{81} \mathrm{Fe}_{19}$ parameters used in the simulations and fitting procedure discussed later were as follows: exchange constant $A=1.3 \times 10^{-11} \mathrm{~J} \mathrm{~m}^{-1}$, saturation magnetization $M_{\mathrm{S}}=7.7 \times 10^{5} \mathrm{~A} \mathrm{~m}^{-1}$ [1], magnetocrystalline anisotropy constant $K=0$, gyromagnetic ratio $\gamma=2.211 \times 10^{5} \mathrm{~m} \mathrm{~A}^{-1} \mathrm{~s}^{-1}$, and dimensionless damping coefficient $\alpha=0.01$. We first imported a relevant magnetic configuration in the form of bitmap (on a grid of $2.5 \mathrm{~nm} \times 2.5 \mathrm{~nm} \times 25 \mathrm{~nm}$ ) and then equilibrated it at a given field. Second, a dynamical simulation was conducted by considering a Gaussian magnetic field pulse of $5 \mathrm{mT}$ amplitude and $2.5 \mathrm{ps}$ full width at half maximum along the $z$-direction (perpendicular to the film plane). We recorded 512 values of magnetization vectors for each grid pixel at 20 ps time steps. The perpendicular component of magnetization was then logged as a function of $x, y$ and the time step. Subsequently, a fast Fourier transformation (FFT) was performed on the magnetization of each grid point along the time axis to obtain the resonance spectrum. The absorbed power for each grid value was obtained by squaring the absolute value of FFT amplitude. The analysis provided a map of spin-precessional amplitudes at a selected frequency. The power 


\section{$+\mathbf{X}$}
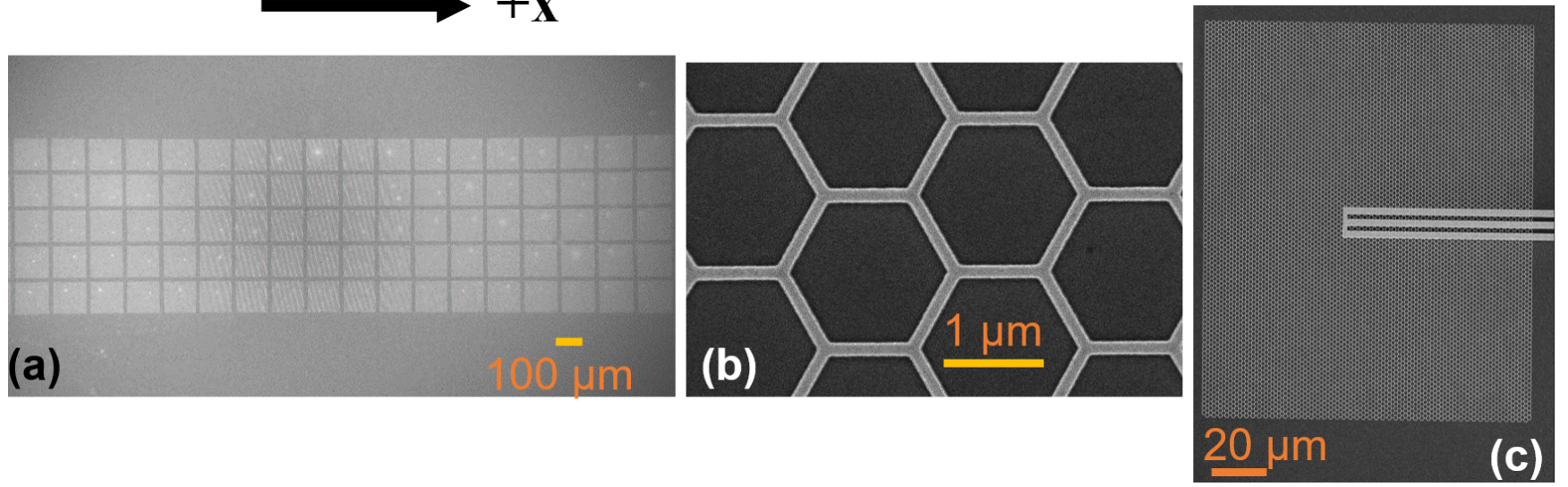

FIG. S1. Representative SEM images of ((a)-(b)) Sample-A and (c) Sample-B. The bright and dark regions in (a) and (b) represent Permalloy and silicon substrate respectively. In (c) the integrated CPW is apparent in addition on the right.

\section{(a)}
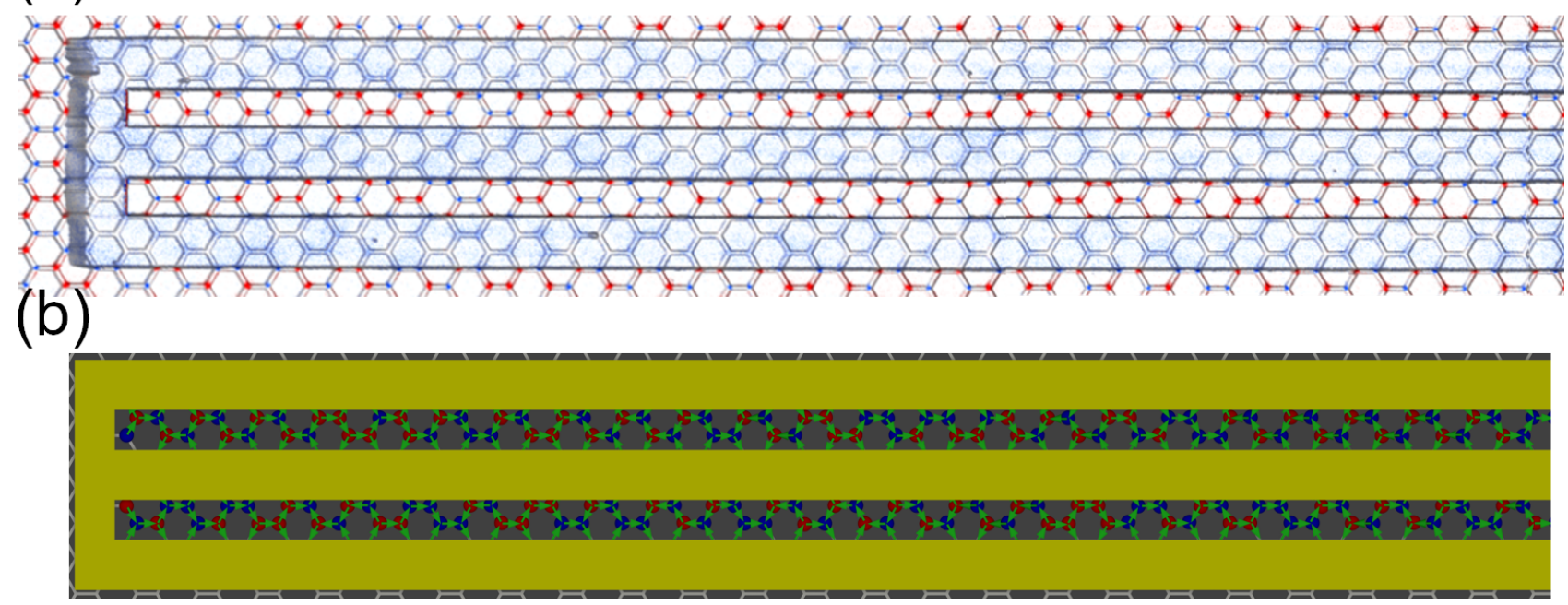

FIG. S2. (a) Magnetic force microscopy image of Sample-B after a field protocol of $-91.2 \mathrm{mT} \rightarrow 44.1 \mathrm{mT} \rightarrow 0 \mathrm{mT}$. Here the blue and red colors represent $+q$ and $-q$ charges, respectively. The scale bar corresponds to $1 \mu$ m. Vertices below the metallic CPW produce a very weak stray-field signal. (b) Cartoon representation of magnetization maps determined using MFM and BLS measurements, respectively. The blue and red colors depict $+q$ and $-q$ charges, respectively. In (a) and (b) the integrated CPW is seen.

spectra were obtained by integrating over all grid points for each frequency step. We show spatial distributions $(x, y$ maps) of square of spin-precessional amplitudes. 


\section{C1}

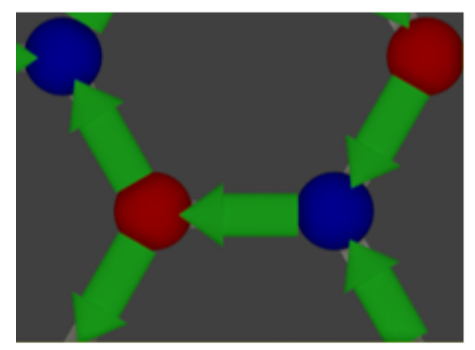

\section{Low High}

$9.3 \mathrm{GHz} \quad 9.4 \mathrm{GHz} \quad 9.65 \mathrm{GHz}$
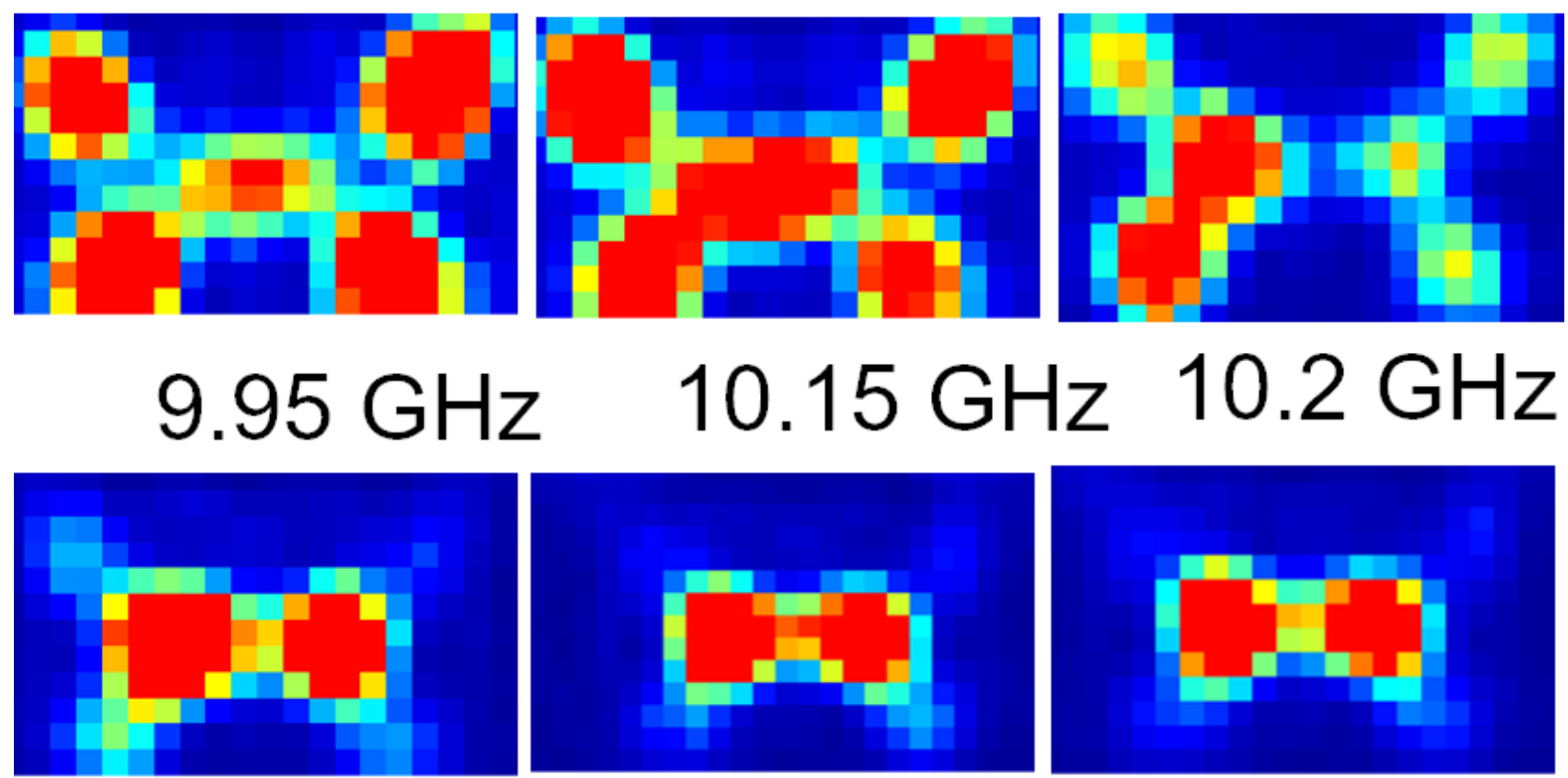

FIG. S3. Spatially resolved BLS intensity maps for various frequencies for magnetization configuration C1. Red and blue colors represent high and low BLS intensities, respectively. 


\section{C2}

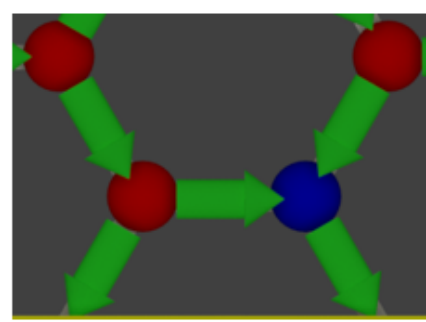

\section{Low High}

$10.4 \mathrm{GHz} \quad 10.85 \mathrm{GHz} 11.05 \mathrm{GHz}$
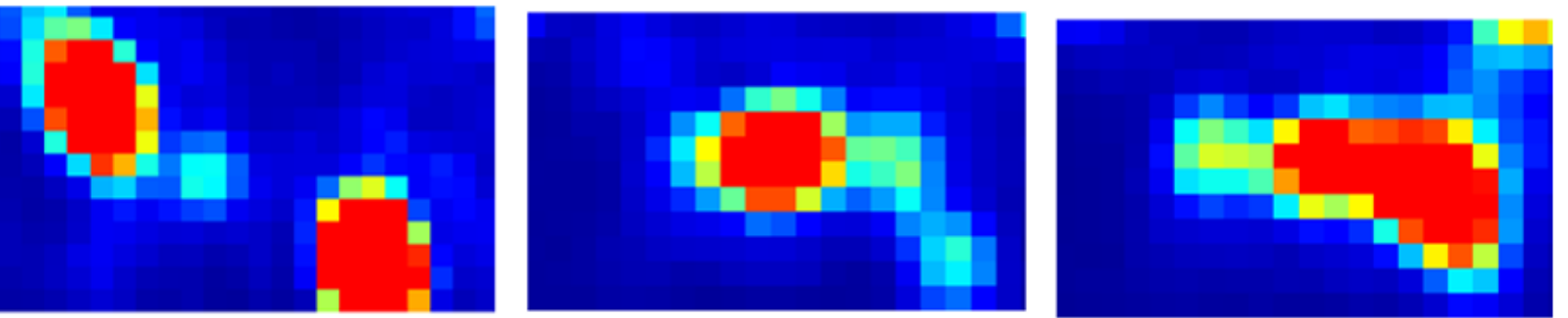

$11.15 \mathrm{GHz} 11.2 \mathrm{GHz} 11.45 \mathrm{GHz}$
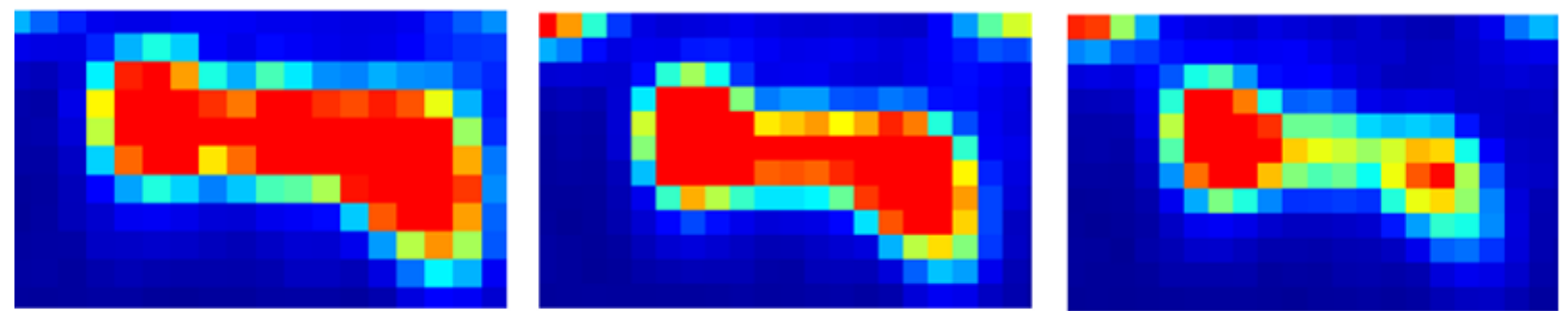

\section{$9.5 \mathrm{GHz}$}

$9.3 \mathrm{GHz}$

$9.2 \mathrm{GHz}$
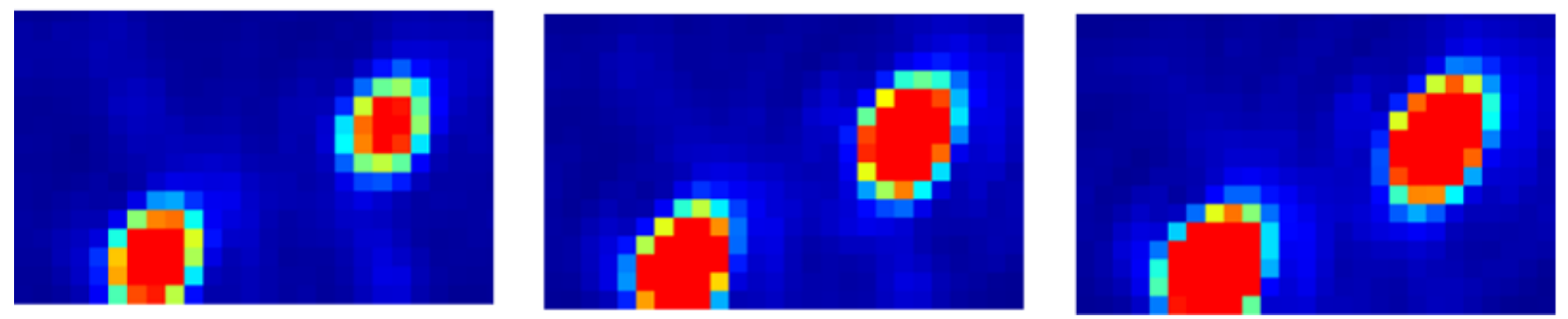

FIG. S4. Spatially resolved BLS intensity maps for various frequencies for magnetization configuration C2. Red and blue colors represent high and low BLS intensities, respectively. 


\section{C3}

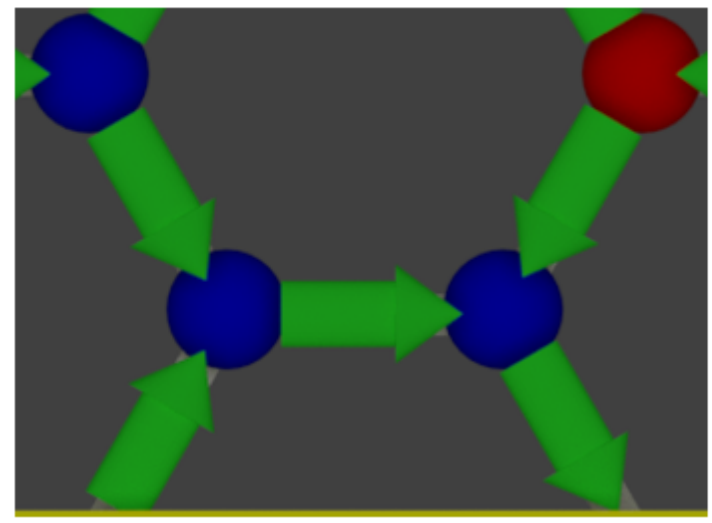

\section{Low High}

\section{$10.4 \mathrm{GHz} 10.85 \mathrm{GHz} 11.2 \mathrm{GHz}$}
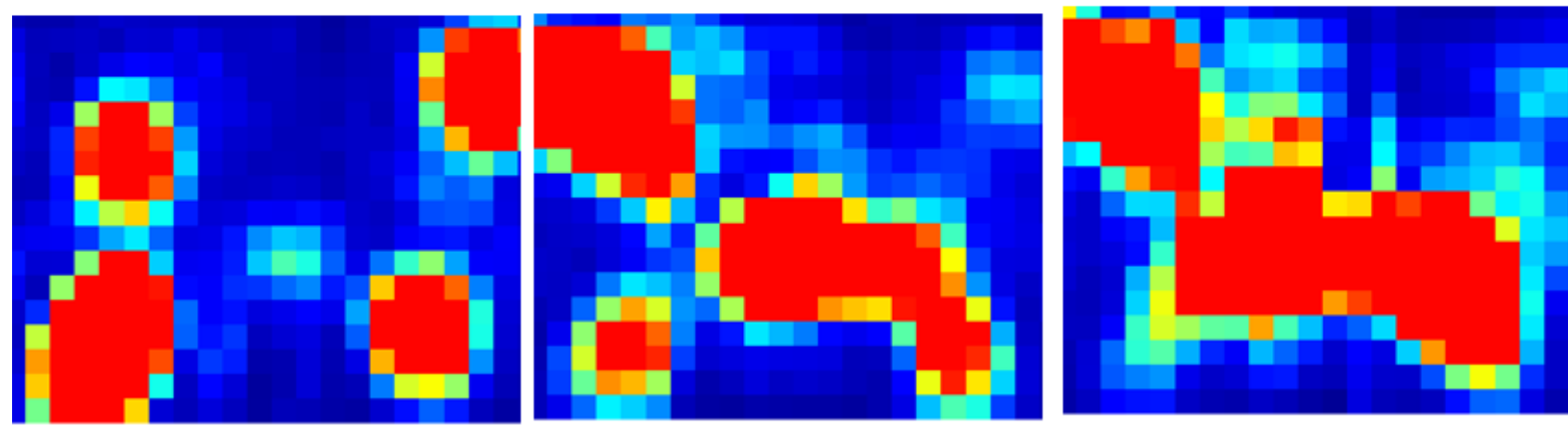

\section{$11.5 \mathrm{GHz}$}

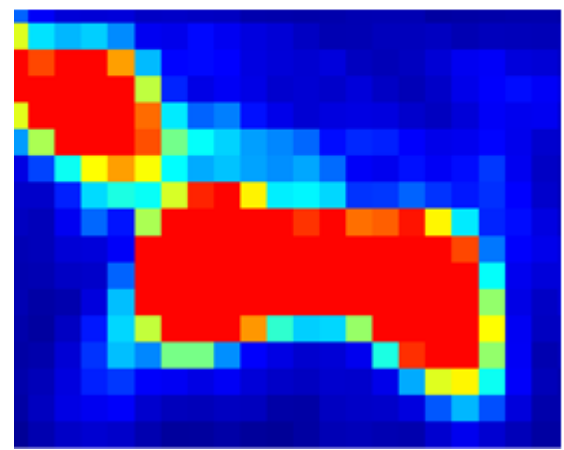

FIG. S5. Spatially resolved BLS intensity maps for various frequencies for magnetization configuration C3. Red and blue colors represent high and low BLS intensities, respectively.. 


\section{C4}

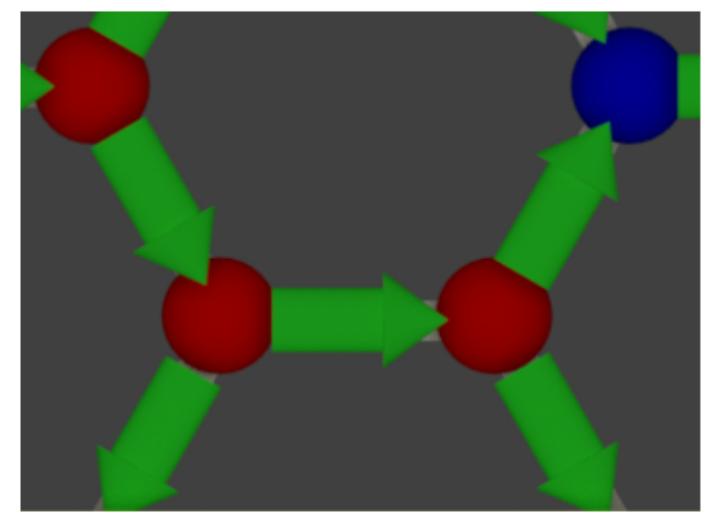

\section{Low High}

$10.8 \mathrm{GHz}$

$11.3 \mathrm{GHz}$

$11.4 \mathrm{GHz}$
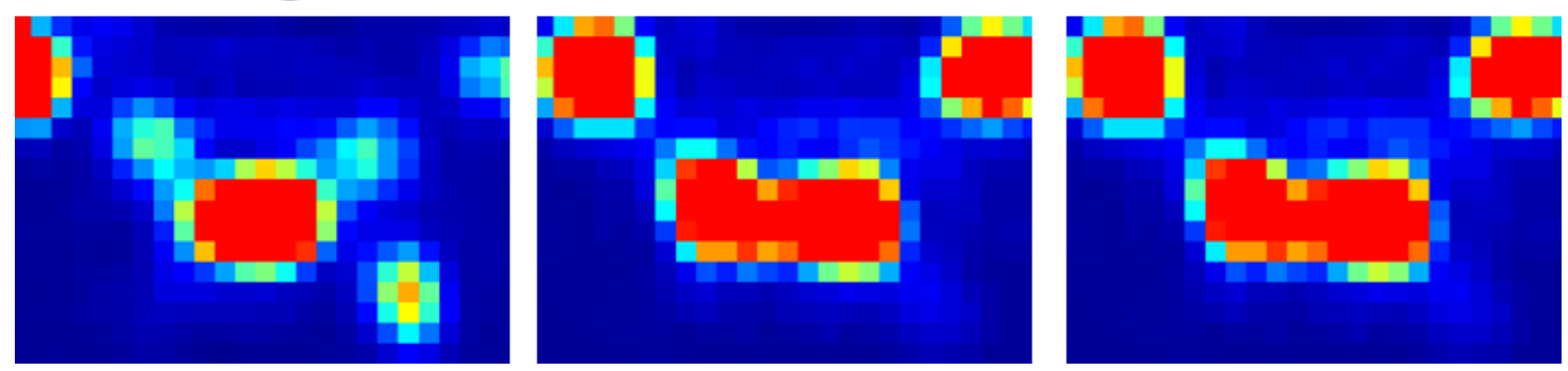

FIG. S6. Spatially resolved BLS intensity maps for various frequencies for magnetization configuration C4. Red and blue colors represent high and low BLS intensities, respectively. 


\section{C5}
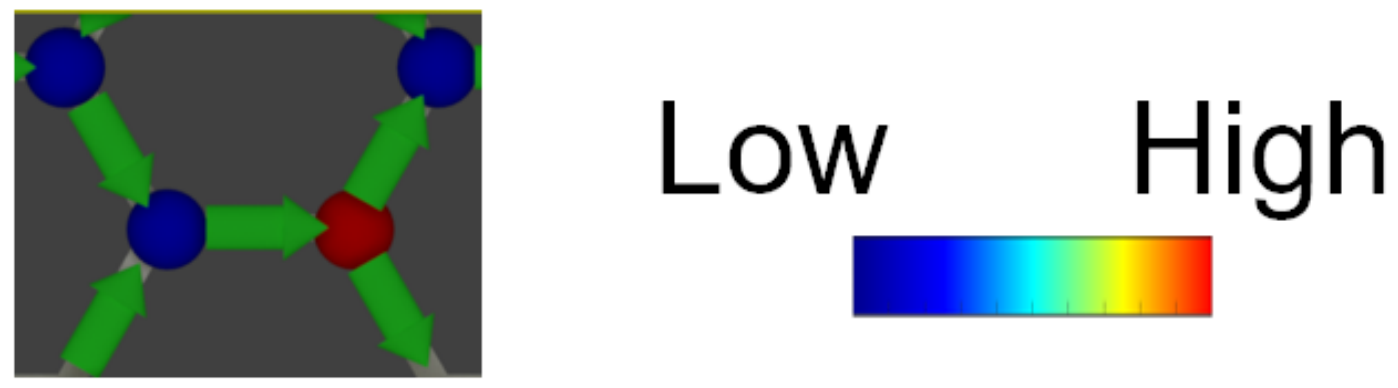

$9.5 \mathrm{GHz} \quad 10.15 \mathrm{GHz} \quad 10.3 \mathrm{GHz}$
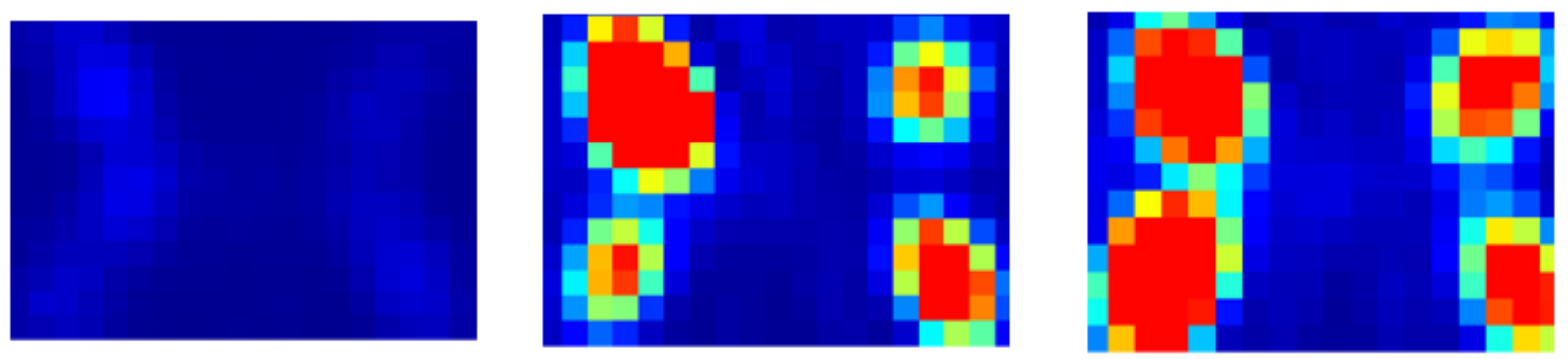

$10.5 \mathrm{GHz} \quad 10.85 \mathrm{GHz} \quad 10.65 \mathrm{GHz}$
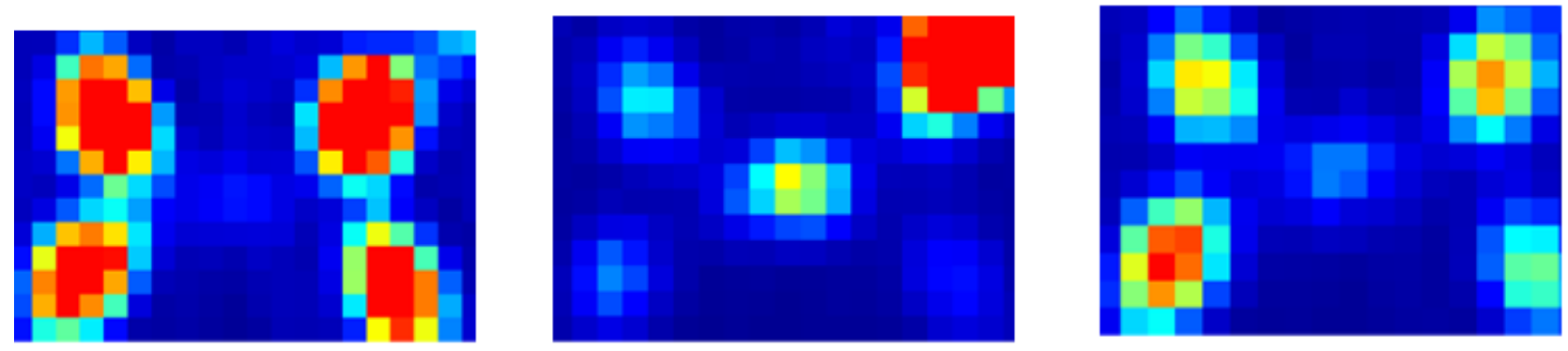

$11.35 \mathrm{GHz} 11.4 \mathrm{GHz} \quad 11.6 \mathrm{GHz}$
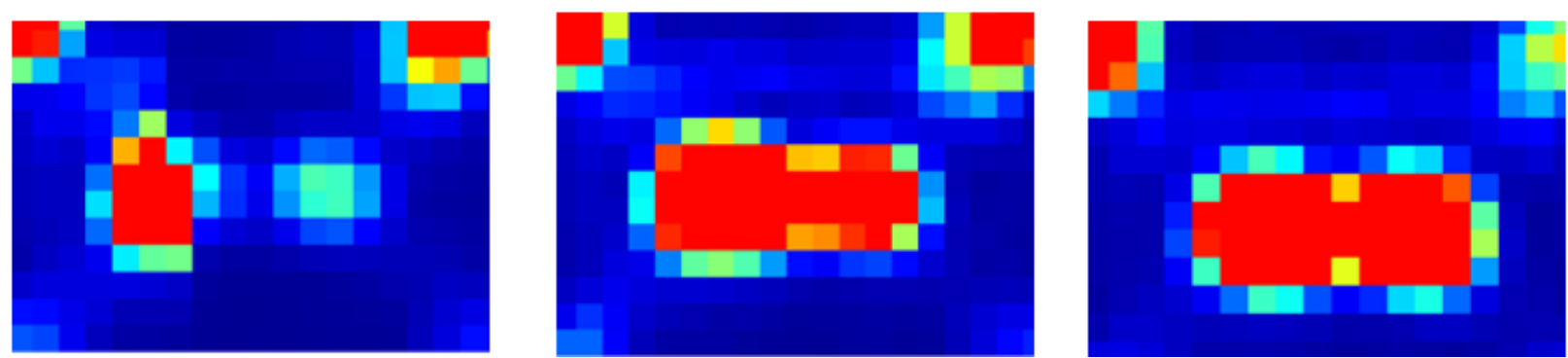

FIG. S7. Spatially resolved BLS intensity maps for various frequencies for magnetization configuration C5. Red and blue colors represent high and low BLS intensities, respectively. 


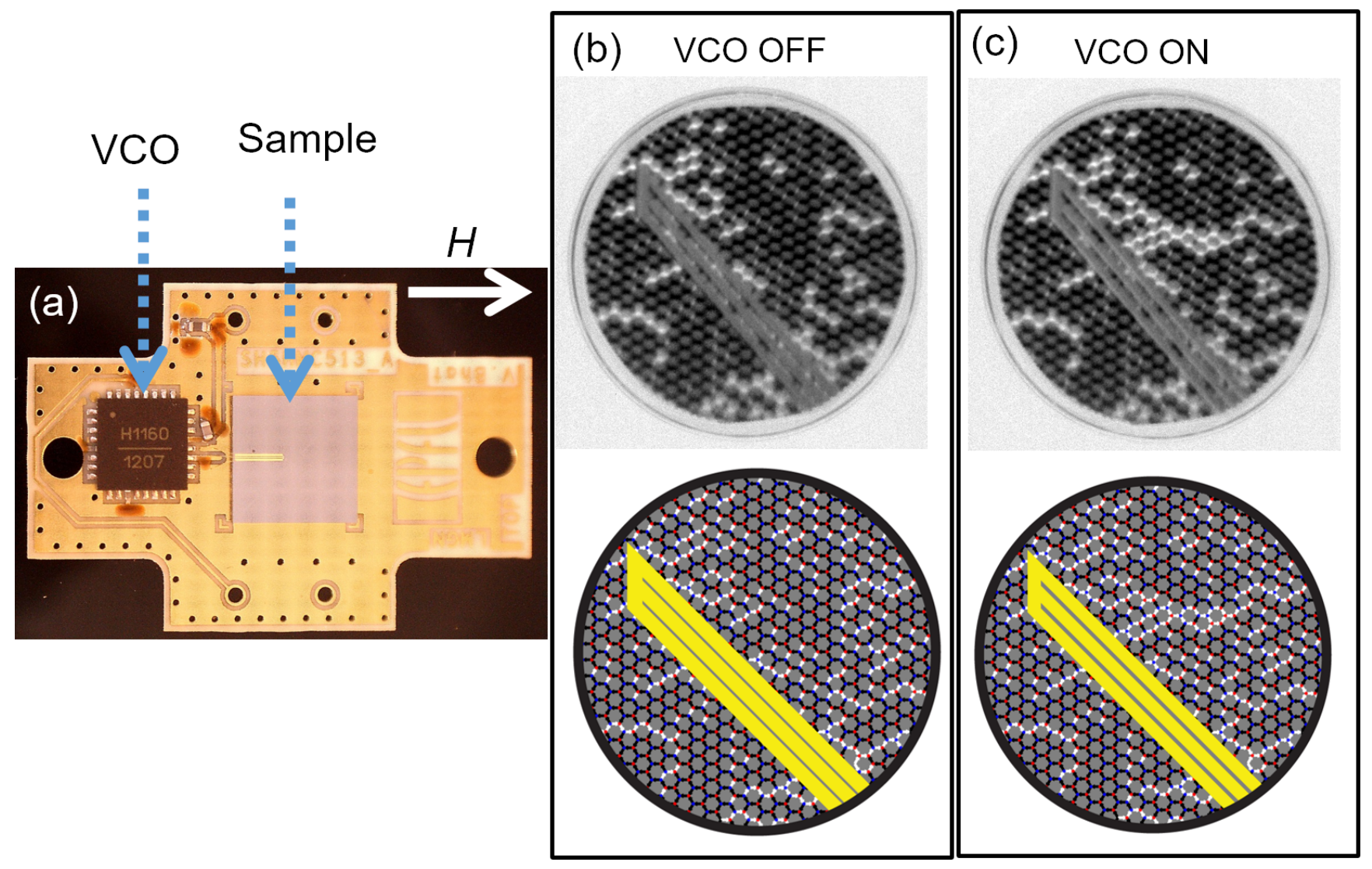

FIG. S8. (a) The printed circuit board with a voltage controlled oscillator (VCO) mounted. VCO supplied the RF power ranging from $7.6 \mathrm{GHz}$ to $9.6 \mathrm{GHz}$. The sample is sitting in the center. XMCD image (top row) together with its cartoon representation (bottom row) depicting magnetic state of the nanobars (b) before (c) and after turning ON the VCO at 9.3 $\mathrm{GHz}$. The red and blue color dot represents vertices with two-in-one-out and two-out-one-in configurations, respectively. 
The research was supported by the Swiss National Science Foundation via Grant No. 163016. V.S. Bhat acknowledges support from the foundation for Polish Science through the IRA Programme financed by EU within SG OP Programme. Part of this work was performed at the Surface/Interface Microscopy (SIM) beamline of the Swiss Light Source (SLS), Paul Scherrer Institut (PSI), Villigen Switzerland.

* vbhat@magtop.ifpan.edu.pl; dirk.grundler@epfl.ch

[1] R. Wang, Geometrical magnetic frustration and demagnetization of artificial spin ice, Ph.D. thesis, The Pennsylvania State University (2007). 